\title{
Effect of deformation on gamow-teller strength and electron capture cross-section for isotopes of chromium
}

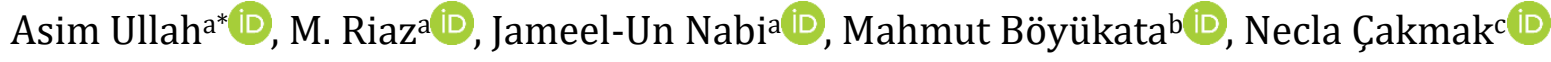 \\ a GIK Institute of Engineering Sciences and Technology, Swabi, KPK, Topi 23640, PAKISTAN, \\ b Kirıkkale University, Science and Arts Faculty, Physics Department, Kırlkkale, 71450, TURKEY, \\ c Karabük University, Science Faculty, Department of Physics, Karabük, 78050, TURKEY,
}

\section{A R T I C LE IN F O}

\section{Article history:}

Received 30 October 2019

Received in revised form 24 April 2020

Accepted 24 April 2020

\section{Keywords:}

Gamow-Teller strength

Electron capture cross-section

pn-QRPA

Deformation parameter

Chromium isotopes

\begin{abstract}
A B S T R A C T
In this work, we explore the role of deformation parameter $(\beta)$ on the calculated Gamow-Teller (GT) strength distributions and electron capture cross-sections (ECC) for $46,48,50 \mathrm{Cr}$ isotopes within the formalism of the proton neutron-quasi-particle random phase approximation (pn-QRPA). Three different $\beta$ parameters were used in the present study. Two of them were calculated by using the interacting boson model (IBM) and the macroscopic-microscopic (Mac-mic) models. The third one is the experimental $\beta$ values obtained by employing its relation with the experimental $\mathrm{B}(\mathrm{E} 2) \uparrow$ values. The GT strength distributions were widely dispersed among all the daughter states of the given isotopes. They were found to have inverse relation with $\beta$ parameter i.e decreasing with increasing the $\beta$ value. The ECC were computed as a function of $\beta$ parameter and the results suggest that the calculated ECC decreased with decreasing value of the $\beta$ for the selected cases.
\end{abstract}

(C) 2020. Turkish Journal Park Academic. All rights reserved.

\section{Introduction}

The death of stars is commonly known as called supernova explosion. Study of such phenomenon can be used to explore our Universe. The electron capture (EC) and $\beta$-decay are keys to decipher the mysterious mechanism of the late stages of stellar evolution [1]. These weak decay processes also play essential role in estimating the pre-supernova core composition as well as synthesis of neutron-rich (massive) nuclei [1-2]. The EC process lowers the electron degenerate pressure and eventually causes the core collapse of the massive star. Thus, the collapse began through EC on iron regime nuclei [3]. In the start of the collapse i.e. at low stellar densities $\left(\sim 10^{10} \mathrm{gcm}^{-3}\right)$ and low temperature $(0.3-0.8 \mathrm{MeV})$, when the $Q$ value and chemical potential of electrons have comparable magnitudes, the EC rates are sensitive to associated GT strength. For higher values of temperature and densities, the chemical potential surpasses the $Q$ value and then the EC rates are largely dictated by the total GT strength. Therefore, the computation of the EC rates and GT strength distribution in the stellar matter is essential requirement.
Fuller, Fowler, and Newman [1] performed the pioneering calculation by tabulating the weak interaction rates employing the independent-particle model (IPM) with the help of available experimental data for astrophysical applications. Weak rates were tabulated for nuclei in mass range $21 \leq \mathrm{A} \leq 60$. Later large-scale shell-model (LSSM) [4] was utilized to improve $\beta^{ \pm}$decay, positron and electron capture rates with the help of calculated GT strength distributions for various nuclei in the mass range 45-65. Based on these revised weak interaction rates, the presupernova phases of heavy mass stars were investigated in Ref. [5]. It was concluded that GT strength distributions and EC rates for $f p$-shell nuclei have significant impact on the presupernova evolution of massive stars. In recent past the proton neutron-quasi-particle random phase approximation (pn-QRPA) model was employed for the investigation of the deformation on the computed ECC [6] and EC rates in stellar matter for the $f p$-shell nuclei [7]. These EC rates were found larger than the past computations when compared at high stellar temperatures.

Recently, pn-QRPA and IBM-1 have been used for the investigation of some nuclear properties for some nuclei as

\section{* Corresponding author.}

E-mail address: asimullah844@gmail.com

ORCID: 0000-0002-2653-310X (Asım.ullah), 0000-0003-4521-4259 (Muhammed.riaz), 0000-0002-8229-8757 (Jameel-un.nabi), 0000-0002-8065-9993

(Mahmut.böyükata), 0000-0001-5989-6663 (Necla.çakmak) 
reported in Refs. [8-10]. In the current paper, the pn-QRPA model with deformed basis is utilized to calculate the GT strength and ECC on even-even $46,48,50 \mathrm{Cr}$ isotopes. Furthermore, the impact of the deformation parameter $(\beta)$ on the calculated GT strength and ECC for the selected cases is explored. The next section describes the essential theoretical framework used in our calculation. We display our results in Section-III. Conclusions are stated in Section-IV.

\section{Model Description}

The pn-QRPA model was used to calculate the GT (chargechanging) strength distribution and associated ECC on the selected chromium isotopes in the stellar matter. The following Hamiltonian was considered

$\mathrm{H}^{\mathrm{QRPA}}=\mathrm{H}^{\mathrm{sp}}+\mathrm{V}_{\mathrm{GT}}^{\mathrm{ph}}+\mathrm{V}_{\mathrm{GT}}^{\mathrm{pp}}+\mathrm{V}^{\text {pair }}$,

where $\mathrm{H}^{\mathrm{sp}}$ is the single particle Hamiltonian, $\mathrm{V}_{\mathrm{GT}}^{\mathrm{ph}}$ and $\mathrm{V}_{\mathrm{GT}}^{\mathrm{pp}}$ are the particle-hole GT force and particle-particle GT force respectively. The last term $\mathrm{V}^{\text {pair }}$ stands for the pairing force for which the BSC approximation was considered. The wave functions and single particle energies were computed within the formalism of Nilsson model [11], in which the $\beta$ value was incorporated. The particle-particle and particle-hole parameter were optimized such that the experimental halflives of the nuclei were reproduced. The Ikeda sum rule [12] was accomplished. The Nilsson potential parameters (NPP) were taken from Ref. [13] and $\hbar \omega=41 \mathrm{~A}^{1 / 3}$ was taken as oscillator constant for both neutrons and protons. Q-values were taken from Ref. [14] and the traditional relation $\Delta_{\mathrm{p}}=$ $\Delta_{\mathrm{n}}=12 / \sqrt{\mathrm{A}}(\mathrm{MeV})$ was considered for estimation of pairing gaps.

The electron capture (EC) and positron decay (PD) weakrates from parent state " $\mathrm{m}$ " to daughter state " $\mathrm{n}$ " are given by

$\lambda_{\mathrm{mn}}^{\mathrm{EC}(\mathrm{PD})}=\ln 2 \frac{\mathrm{f}_{\mathrm{mn}}^{\mathrm{EC}(\mathrm{PD})}\left(\mathrm{T}, \rho, \mathrm{E}_{\mathrm{f}}\right)}{\mathrm{D} / \mathrm{B}_{\mathrm{mn}}}$,

where $B_{m n}$ is the nuclear reduced transition probability and is given by

$\mathrm{B}_{\mathrm{mn}}=\mathrm{B}(\mathrm{F})_{\mathrm{mn}}+\left(\mathrm{g}_{\mathrm{A}} / \mathrm{g}_{\mathrm{v}}\right)^{2} \mathrm{~B}(\mathrm{GT})_{\mathrm{mn}}$,

The values of $\mathrm{D}$ and $\mathrm{g}_{\mathrm{A}} / \mathrm{g}_{\mathrm{V}}$ were taken as 6143s [15] and -1.254 [16], respectively.

The reduced Fermi $\left(\mathrm{B}(\mathrm{F})_{\mathrm{mn}}\right)$ and $\mathrm{GT}\left(\mathrm{B}(\mathrm{GT})_{\mathrm{mn}}\right)$ transition probabilities were calculated using the following reduced transition probabilities:

$\mathrm{B}(\mathrm{F})_{\mathrm{mn}}=\frac{1}{2 \mathrm{~J}_{\mathrm{m}}+1}\left|\left\langle\mathrm{n}\left\|\sum_{\mathrm{k}} \mathrm{t}_{+}^{\mathrm{k}}\right\| \mathrm{m}\right\rangle\right|^{2}$,

$\mathrm{B}(\mathrm{GT})_{\mathrm{mn}}=\frac{1}{2 \mathrm{~J}_{\mathrm{m}}+1}\left|\left\langle\mathrm{n}\left\|\sum_{\mathrm{k}} \mathrm{t}_{+}^{\mathrm{k}} \sigma^{\rightarrow \mathrm{k}}\right\| \mathrm{m}\right\rangle\right|^{2}$,

here $J_{m}$ shows the total spin of the parent state $|m\rangle, \sigma^{\rightarrow k}$ is the Pauli spin matrix and $t_{+}^{k}$ refer to the iso-spin raising operator.

The computation of ECC is governed by the weak-interaction Hamiltonian, given by
$\widehat{\mathrm{H}}_{\omega}=\frac{\mathrm{G}_{\mathrm{F}} \cos \theta_{\mathrm{c}}}{\sqrt{2}} \mathrm{j}_{\mu}^{\text {lept }} \hat{\mathrm{J}}^{\mu}$

The terms $\theta_{c}$ and $G_{F}$ in the above equation, stands for Cabibbo angle and Fermi coupling constant, respectively. The $\hat{J}^{\mu}$ and $\mathrm{j}_{\mu}^{\text {lept }}$ are the hadronic and leptonic currents, respectively, given by

$\mathrm{j}_{\mu}^{\text {lept }}=\bar{\psi}_{\mathrm{v}_{\mathrm{e}}}(\mathrm{x}) \gamma_{\mu}\left(1-\gamma_{5}\right) \psi_{\mathrm{v}_{\mathrm{e}}}(\mathrm{x})$

$\hat{\mathrm{J}}^{\mu}=\bar{\psi}_{\mathrm{p}}(\mathrm{x}) \gamma_{\mu}\left(1-\mathrm{C}_{\mathrm{A}} \gamma_{5}\right) \psi_{\mathrm{n}}(\mathrm{x})$

where $\psi_{v_{\mathrm{e}}}(\mathrm{x})$ show the spinor operator. The terms $\gamma_{\mu}$ and $\gamma_{5}$ are stands for Dirac $\gamma$-matrices. $C_{A}$ is a constant which comes as a consequence of the internal structure of the hadrons. Our main goal was to compute the ECC which is based on nuclear matrix elements from parent state $|m\rangle$ to daughter state $|n\rangle$ and is described by;

$\left\langle\mathrm{n}\left\|\widehat{\mathrm{H}}_{\omega}\right\| \mathrm{m}\right\rangle=\frac{\mathrm{G}}{\sqrt{2}} \mathrm{l}^{\mu} \int \mathrm{d}^{3} \mathrm{xe}^{-\mathrm{iq} \cdot \mathrm{x}}\left\langle\mathrm{n}\left|\hat{\mathrm{J}}_{\mu}\right| \mathrm{m}\right\rangle$

The term $\mathrm{q}$ in the equation refers to the three-momentum transfer and $l^{\mu} e^{-i q \cdot x}$ stands for the leptonic matrix element which was employed in matrix elements calculation $[17,18]$. We applied the low momentum transfer approximation $q \rightarrow 0$ in this work. Such approximation enables the GT operator $\left(\mathrm{GT}^{+}=\sum_{\mathrm{i}} \tau_{\mathrm{i}}^{+} \sigma_{\mathrm{i}}\right)$ to contribute dominantly to the total ECC [18]. The total ECC in the stellar condition in terms of incident electron energy $\left(E_{e}\right)$ may then be computed using the following equation

$$
\begin{aligned}
\sigma\left(E_{e}, T\right) & =\frac{G_{F}^{2} \cos ^{2} \theta_{c}}{2 \pi} \sum_{m} F\left(Z, E_{e}\right) \frac{\left(2 J_{m}+1\right) \exp \left(\frac{-E_{m}}{k T}\right)}{G(A, Z, T)} \\
& \times \sum_{J, f}\left(E_{e}-Q+E_{m}-E_{m}\right)^{2} \frac{\left|\left\langle m\left|G T^{+}\right| n\right\rangle\right|^{2}}{\left(2 J_{m}+1\right)}
\end{aligned}
$$

The Fermi function denoted by $\mathrm{F}\left(\mathrm{Z}, \mathrm{E}_{\mathrm{e}}\right)$ in the above equation was calculated using the recipe of Ref. [19]. To compute the famous nuclear partition function $G(Z, A, T)$, we used the prescription recently introduced in Refs. [20,21]. This recipe is believed to give a more realistic estimate of the partition functions appearing in Eq. (10). The final term in Eq. (10) corresponds to the nuclear matrix elements of $\left(\mathrm{GT}^{+}=\right.$ $\sum_{\mathrm{i}} \tau_{\mathrm{i}}^{+} \sigma_{\mathrm{i}}$ ) operator between final and initial states.

The underlying theme of this current work was to study the influence of the $\beta$ parameter on the calculated GT strength distribution and ECC for the given $\mathrm{Cr}$ isotopes. To accomplish the objective, three different $\beta$ values listed in Table 1 were considered. Two of $\beta$ values were calculated employing theoretical models: Macroscopic-microscopic (mac-mic) model [22] and IBM-1 [23].

The Mac-mic model computed the electric quadrupole moment $\left(Q_{2}\right)$. We used the value of $Q_{2}$ in the following equation to determine the deformation parameter:

$\beta=\frac{125 \mathrm{Q}_{2}}{1.44 \mathrm{~A}^{2 / 3 \mathrm{Z}}}$

For the calculation of deformation parameters within the IBM1 model; first the energy levels of the given nucleus were calculated with a simplified model Hamiltonian by fitting its parameters taken as constants;

$\widehat{H}=\epsilon \hat{n}_{d}+\kappa \hat{Q} \cdot \hat{Q}+\kappa^{\prime} \hat{L} \cdot \hat{L}$ 
where $\hat{n}_{d}$ and $\hat{L}$ are the boson-number and the angular momentum terms, respectively. Other term is the quadrupole operator given by

$$
\hat{Q}_{\mu}=\left[d^{\dagger} \times \tilde{s}+s^{\dagger} \times \tilde{d}\right]_{\mu}^{(2)}+\chi\left[d^{\dagger} \times \tilde{d}\right]_{\mu}^{(2)},
$$

where $\chi$ is the fourth parameter and four parameters in total were fitted for given $\mathrm{Cr}$ isotopes. Later, the potential energy surfaces of the given nucleus was plotted as function of deformation parameters to calculate the $\beta$ value. The potential energy surface can be formulated [24-28] from the given model Hamiltonian Eq. (12) as following;

$$
\begin{aligned}
V(\beta, \gamma) & =N \frac{\beta^{2}}{1+\beta^{2}}\left[\epsilon+\kappa^{\prime} 6+\kappa\left(\frac{5+\left(1+\chi^{2}\right) \beta^{2}}{\beta^{2}},\right.\right. \\
& \left.\left.+\frac{N-1}{N} \frac{\frac{2 \chi^{2} \beta^{2}}{7}-4 \sqrt{\frac{2}{7}} \chi \beta \cos (3 \gamma)+4}{1+\beta^{2}}\right)\right],
\end{aligned}
$$

where it is seen that the $\epsilon, \kappa, \kappa^{\prime}$, and $\chi$ parameters are the common constants given in Eqs. (12-13), $N$ is the boson number of the nucleus and the $\beta, \gamma$ are called as deformation parameters having same role Bohr-Mottelson model [29].

The experimental $\beta$ values were computed by employing the following equation

$\beta=\frac{4 \pi}{3 \mathrm{ZR}_{0}^{2}} \times\left[\frac{\mathrm{B}(\mathrm{E} 2) \uparrow}{\mathrm{e}^{2}}\right]^{1 / 2}$

where $\mathrm{R}_{0}{ }^{2}=0.0144 \mathrm{~A}^{2 / 3} \mathrm{~b}$ and $\mathrm{B}(\mathrm{E} 2) \uparrow$ is in units of $\mathrm{e}^{2} \mathrm{~b}^{2}$. The reduced electric quadrupole transition probability B(E2) $\uparrow$ was taken directly from the National Nuclear Data Center (NNDC) [30].

\section{Results and Discussions}

In Table 1, fitted parameters of the simplified Hamiltonian given in Eq. (12) are listed and these parameters are also taken as the constant in the energy surface formalism. The units of $\epsilon, \kappa$, and $\kappa^{\prime}$ parameters are in $\mathrm{keV}$ and $\chi$ is dimensionless.

Table 1. The fitted parameters (keV) given as constant in Eqs. (1213) and $\chi$ is dimensionless.

\begin{tabular}{|c|c|c|c|c|}
\hline \hline Isotopes & $\epsilon$ & $\kappa$ & $\kappa^{\prime}$ & $\chi$ \\
\hline${ }^{46} \mathrm{Cr}$ & 0.8742 & -0.0485 & - & -0.45 \\
\hline${ }^{48} \mathrm{Cr}$ & 1.5155 & -0.2333 & -0.0687 & -1.02 \\
\hline${ }^{50} \mathrm{Cr}$ & 1.2002 & -0.1565 & -0.0386 & -0.5 \\
\hline
\end{tabular}

The energy spectra including the calculated and experimental energy levels of the given isotopes are plotted in Figure 1 . The calculated levels are marked by the black solid line and the experimental ones [30] given with the blue dashed lines. As seen from this figure the calculated energy levels with IBM-1 are quite close to the experimental ones [30].

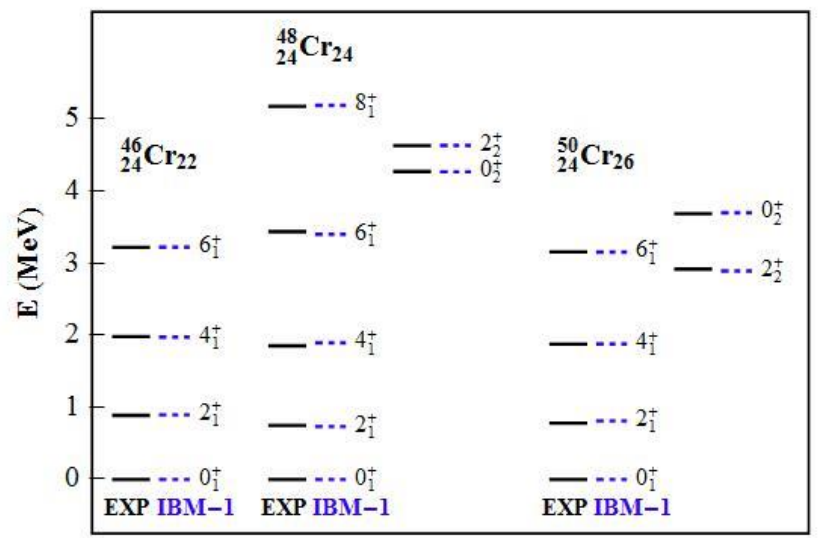

Figure 1. The experimental [29] and the calculated energy levels of selected $\mathrm{Cr}$ isotopes.

In Figure 2, the potential energy surfaces of IBM-1 are plotted in terms of $\beta$ parameter $(\gamma=0)$ for given $\mathrm{Cr}$ isotopes. The $\beta$ parameters of each isotope can be calculated from the minimum point of the energy surfaces. These values are listed in Table 2. As seen from Figure $2,{ }^{46} \mathrm{Cr}$ has a spherical shape since this parameter is zero whereas ${ }^{48} \mathrm{Cr}$ and ${ }^{50} \mathrm{Cr}$ have deformed prolate shape. The even-even ${ }^{46} \mathrm{Cr},{ }^{48} \mathrm{Cr}$, and ${ }^{50} \mathrm{Cr}$ isotopes display shape changing from spherical to prolate along the isotopic chain for $22 \leq \mathrm{N} \leq 26$.

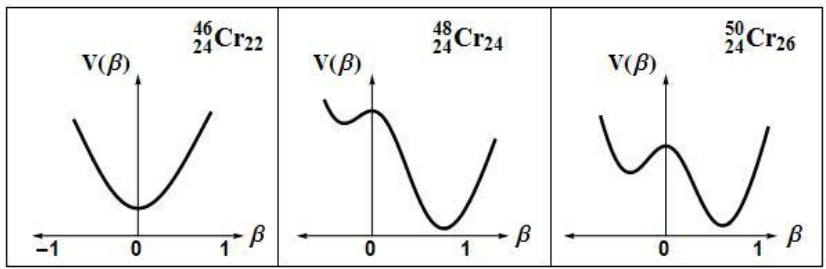

Figure 2. The energy surfaces as function of $\beta$ parameter for the given $\mathrm{Cr}$ isotopes.

Table 2 summarizes the centroid and total GT strength values, computed from the calculated GT strength distributions for the selected $\mathrm{Cr}$ isotopes. The third column shows the values of $\beta$ parameter computed employing the respective models given in second column of Table 2 . The previous section described how these values were calculated. The table reflects that the total GT strength lowers as the mass number increases. This outcome can be attributed to the fact that the EC process turns more difficult for higher neutron number. The centroid value decreases for heavier cases because the neutron excess increases. The table also shows the effect of deformation on the computed GT strength. It is noted that for each isotope, with increase in the value of $\beta$ the calculated GT strength decreases. This is an interesting outcome, but we cannot generalize this statement which requires further investigation currently in progress. 
Table 2. The computed centroid and total GT strength values for the obtained $\beta_{2}$ parameter for the given $\mathrm{Cr}$ isotopes.

\begin{tabular}{|c|c|c|c|c|}
\hline \hline İsotopes & Model & $\begin{array}{c}\text { Deformation } \\
\text { Parameter } \\
\beta\end{array}$ & $\begin{array}{c}\text { Total GT } \\
\text { strength } \\
\sum{ }^{46} \mathrm{Cr}\left(\mathrm{GT}_{+}\right)\end{array}$ & $\begin{array}{c}\text { Centroid } \\
\overline{\mathrm{E}}_{+}(\mathrm{MeV})\end{array}$ \\
\hline \multirow{3}{*}{${ }^{48} \mathrm{Cr}$} & Mac-mic & 0.028 & 9.13 & 12.37 \\
& IBM-1 & 0 & 9.67 & 12.15 \\
& NNDC & 0.288 & 8.97 & 12.76 \\
\hline \multirow{3}{*}{$\mathrm{F} \mathrm{Cr}$} & Mac-mic & 0.23551 & 8.94 & 11.74 \\
& IBM-1 & 0.760 & 8.46 & 12.02 \\
& NNDC & 0.368 & 8.63 & 11.95 \\
& Mac-mic & 0.14124 & 8.58 & 10.78 \\
& IBM-1 & 0.586 & 5.75 & 11.14 \\
& NNDC & 0.29 & 7.66 & 10.94 \\
\hline
\end{tabular}

Figure 3 displays the computed ECC on ${ }^{46,48,50} \mathrm{Cr}$ as a function of incident electron energy $\left(\mathrm{E}_{\mathrm{e}}\right)$ in the range $(0-30) \mathrm{MeV}$. It is found that for each $\mathrm{Cr}$ isotope the ECC increases with increasing incident electron energy $\left(E_{e}\right)$ because of the term $\left(E_{e}-Q+E_{m}-E_{m}\right)^{2}$ in Eq. (10). It is also noted the ECC increases exponentially in the energy range (1-1.5) $\mathrm{MeV}$. When the temperature of the core rises from $0.5 \mathrm{MeV}$ to 1.0 MeV, there is a prominent increase in the calculated ECC value up to a factor 50. Configuration mixing and thermal unblocking of states could be cited as probable sources for this increment [31]. With a further increase in temperature from $1.0 \mathrm{MeV}$ to $1.5 \mathrm{MeV}$, the increment in the computed ECC is very small (less than a factor of 2). This is because majority of the transitions are already unblocked at such high temperatures. The steep increase may be attributed to the behavior of the computed GT strength distribution. The centroid of the GT strength distribution shifts by few $\mathrm{MeV}$ with rise in temperature. The nuclear partition functions also increase with rise in core temperature. These factors are responsible for the change in behavior of the ECC.

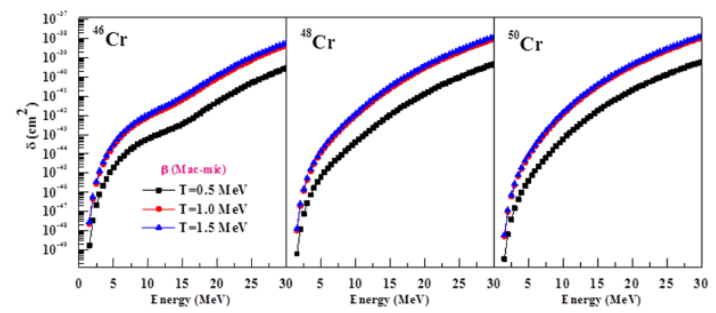

Figure 3. Calculated ECC on ${ }^{46,48,50} \mathrm{Cr}$ as a function of incident electron energy for different values of temperature at $\beta$ parameter (Mac-mic).

Lastly we investigated the impact of the $\beta$ parameter on computed ECC for the given isotopes. Figure 4 shows the calculated ECC as a function of the $\beta$ parameter at a fixed temperature of $1 \mathrm{MeV}$. It can be noted from the figure that the computed ECC on the selected $\mathrm{Cr}$ isotopes decreases with decrease in $\beta$ value and vice versa.

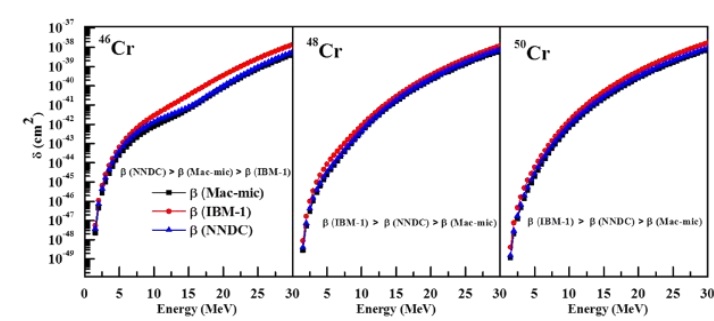

Figure 4. Comparison of the computed ECC on ${ }^{46,48,50} \mathrm{Cr}$ with different $\beta$ values, at fixed temperature $(\mathrm{T}=1 \mathrm{MeV})$.

\section{Summary}

The fundamental theme of the presented study was to explore the influence of the $\beta$ parameter on the calculated GT strength distributions and ECC. To accomplish the goal, we considered three even-even $\mathrm{Cr}$ isotopes and calculated the ECC for these cases at three different temperatures $(0.5,1 \& 1.5 \mathrm{MeV})$. The GT strength distributions obeyed the ISR and were widely dispersed among all the daughter states of the respective nuclei. The centroid and total GT strength values were computed in terms of the $\beta$ parameter. It was found that the total GT strength has inverse relation with the $\beta$ parameter i.e with decreasing $\beta$, the total GT strength increases and vice versa. The computed ECC for the given cases was noted increasing with increase in the core temperature. Comparing the computed ECC at different $\beta$ values, it was found that they increased with rise in the value of $\beta$ parameter. Moreover, the selected ${ }^{46} \mathrm{Cr},{ }^{48} \mathrm{Cr}$, and ${ }^{50} \mathrm{Cr}$ isotopes exhibit shape changing from spherical to prolate. We are studying more isotopes of chromium and hope to report soon on our findings.

\section{Acknowledgment}

J.-U. Nabi would like to acknowledge the support of the Higher Education Commission Pakistan through project numbers 5557/KPK/NRPU/R\&D/HEC/2016 and 9-5(Ph-1-MG-7) PakTurk/R\&D/HEC/2017 and Pakistan Science Foundation through project number PSFTUBITAK/KP-GIKI (02). M. Böyükata would like to acknowledge the support of Higher Education Council of Turkey through project number MEV2019-1745 of project-based international exchange program and the support of the Scientific and Technical Research Council of Turkey (TÜBİTAK) under the project number 119T127. N. Çakmak would like to acknowledge the support of Higher Education Council of Turkey through project number MEV-2018-300 Pak-Turk mobility program.

\section{References}

[1] G. M. Fuller, W. A. Fowler and M. J. Newman, Astrophys. J. Suppl. Ser. 42 (1980).

[2] E. M. Burbidge, G. R. Burbidge, W. A. Fowler and F. Hoyle, Rev. Mod. Phys. 29 (1957) 547.

[3] P. G. Giannaka and T. S. Kosmas, Electron Capture Cross Sections for Stellar Nucleosynthesis, Adv. H. E. Phys. 2015, (2014) 11.

[4] G. Martìnez-Pinedo, K. Langanke, D. J. Dean, Astrophys. J. Suppl. Ser. 126 (2000) 493.

[5] A. Heger, K. Langanke, G. Martìnez-Pinedo, S. E. Woosley, Phys. Rev. Lett. 86 (2001) 1678. 
[6] A. Ullah, J.-U. Nabi and M. Riaz, Int. J. Mod. Phys. D 28 (2019) 2040011.

[7] J.-U. Nabi and M. Riaz, J. Phys. G: Nucl. Part. Phys. 47, (2019) 085201.

[8] J.-U. Nabi, M. Böyükata, Nucl. Phy. A 947 (2016) 182.

[9] J.-U. Nabi, Böyükata, Astrophys Space Sci. 362 (2017) 9.

[10] J.-U. Nabi, M. Ishfaq, M. Böyükata, M. Riaz, Nucl. Phy. A 966 (2017) 1.

[11] S. G. Nilsson, G Nilsson, Mat. Fys. Medd. K. Dan. Vidensk. Selsk. 29 (1955) 16.

[12] K. Ikeda, S. Fujii and J. I. Fujita, Phys. Lett. 3, (1963) 271.

[13] I. Ragnarsson and R. K. Sheline, Phys. Scr. 29, (1984) 385.

[14] G. Audi, F. Kondev, M. Wang, W. Huang, and S. Naimi, Chinese physics C, 41 (2017) 030001.

[15] K. Nakamura, (Particle Data Group): J. Phys. G, Nucl. Part. Phys. 37, (2010) 075021.

[16] J. C. Hardy, and I. S. Towner, Phys. Rev. C 79, (2009) 055502.

[17] N. Paar, G. Col'o, E. Khan, and D. Vretenar, Phys. Rev.C 80 (2009) 055801.

[18] J. D. Walecka, Theoretical nuclear and subnuclear physics, (World Scientific Publishing Company, 2004).

[19] N. Gove and M. Martin, At. Data Nucl. Data Tables, 10 (1971) 205219.

[20] J.-U. Nabi, A. N. Tawfik, N. Ezzelarab, and A. A. Khan, Astrophys. and Space Sc. 361 (2016) 71.

[21] J.-U. Nabi, A. N. Tawfik, N. Ezzelarab, and A. A. Khan, Phys. Scr. 91 (2016) 055301.

[22] P. Moller and J. R. Nix, At. Data Nucl. Data Tables, 26 (1981) 165196.

[23] A. Arima and F. Iachello., Annals of Physics 99 (1976) 253-317.

[24] A. E. L. Dieperink, O. Scholten and F. Iachello, Phys. Rev. Lett. 44 (1980) 1747.

[25] A. E. L. Dieperink, O. Scholten, Nucl. Phys. A 346 (1980) 125.

[26] J. N. Ginocchio, M. W. Kirson, Phys. Rev. Lett. 44 (1980) 1744.

[27] J. N. Ginocchio, M. W. Kirson, Nucl. Phys. A 350 (1980) 31.

[28] P. Van Isacker and J.-Q. Chen, Phys. Rev. C 24 (1981) 684.

[29] A. Bohr and B. R. Mottelson, Nuclear Structure. Volume 2: Nuclear Deformation, World Scientific Publishing, 1998.

[30] National Nuclear Data Center (NNDC), http://www.nndc. bnl.gov/, 2019.

[31] K. Langanke, E. Kolbe, and D. Dean, Phys. Rev. C, 63 (2001) 032801. 